TP Periodica Polytechnica Electrical Engineering and Computer Science

61(1), pp. 48-53, 2017

DOI: 10.3311/PPee.9734

Creative Commons Attribution (i)

RESEARCH ARTICLE

\section{High Frequency Properties of RF Planar Passive Components}

\author{
Alexey Shlykevich ${ }^{1 *}$, Tomas Bystricky ${ }^{1}$, Tomas Blecha ${ }^{1}$
}

Received 22 July 2016; accepted after revision 22 September 2016

\begin{abstract}
This paper describes the different constructions of planar capacitors and inductors which are able to work up to UHF frequencies. Described passive components were manufactured using two different technologies: photolithography on polycarbonate film and on FR-4 substrate, screen printing technology. The high-frequency characteristics of the main electrical parameters (capacitance, inductance, quality factor and impedance) of passive components made using different technologies were measured and compared. Suitable finite element models of capacitors were created using multiplatform software applications COMSOL Multiphysics ${ }^{\circledR}$. Based on obtained data the resonant circuits for wireless operating principle devices were designed and fabricated. Comparison of the calculated values of inductance, capacitance, and resonant frequency with measured data was made.
\end{abstract}

\section{Keywords}

interdigital capacitor (IDC), planar inductor, screen printing technology, polymer films, resonant circuits, resonant frequency

\footnotetext{
${ }^{1}$ Department of Technologies and Measurement,

Faculty of Electrical Engineering, University of West Bohemia,

Pilsen, Czech Republic

*Corresponding author, e-mail: alexeys@ket.zcu.cz
}

\section{Introduction}

The fast growing of the wireless market has created an urgent demand for smaller and cheaper handsets with increased functionality and performance while still meeting the tight constraints for mass production within a short product life cycle. Due to economic aspects, there is a necessity of cheap production of highfrequency components with the specified parameters. In particular, it is also actually for the wireless identification devices.

Many transmission lines and waveguides have been used for microwave and millimeter-wave frequencies. Among them, the rectangular waveguide, coaxial line, and microstrip line are the most commonly used [1]. This article investigates the passive planar components that may be used in particular in passive RFID tags. The authors had a task to make a resonant circuit with an operating frequency of $100 \mathrm{MHz}$. Moreover, its maximum dimensions should not be more than ID1 $(85.6 \times 53.98 \mathrm{~mm})$ format according to ISO / IEC 7810 and technology should provide the minimum cost of the finished product.

Previous research has demonstrated various technologies applicable to the planar components manufacture. This article describes the passive elements manufactured by the photolithography technology as the most wide-spread and screen printing technology [2] which allows maximum reduce the cost of the finished product [3]. Also, screen printing provides a wide range of substrate materials (including paper, fabric) [4].

The basic structure of a capacitor with two electrodes is shown in (a) Fig. 1. Interdigital capacitors (IDC) as lumped circuit elements in RF circuits can be used. IDC represent a multifinger periodic structure as shown in (b) Fig. 1. IDC use the capacitance that occurs across a narrow gap between thin-film conductors. These gaps are essentially very long and folded to use a small amount of area. IDC has a larger size compared with the overlay capacitor (see (a) Fig. 1) with the same capacitance value. The quality factor for such type capacitor is higher than that for overlay capacitors [3 pp. 229-230].

The total capacitance $C_{0}$ of an interdigital structure (see (b) Fig. 1) depends on finger length $l$, finger width $w$ and finger spacing $s$. When $s=w$, capacitance $C_{0}$ can be calculated according to $[4$ p. 60$]$ by the Eq. (1): 


$$
C=\left[\left(\varepsilon_{r}+1\right) / w \cdot l\right] \cdot\left[(N-3) \cdot A_{1}+A_{2}\right](p F)
$$

where, for no ground plane, $\mathrm{A}_{1}=4.409 \times 10^{-6} \mathrm{pF} / \mathrm{mm}$ and $\mathrm{A}_{2}=9.92 \times 10^{-6} \mathrm{pF} / \mathrm{mm}$.

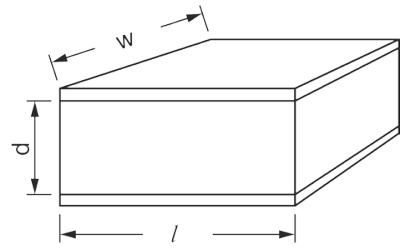

(a)

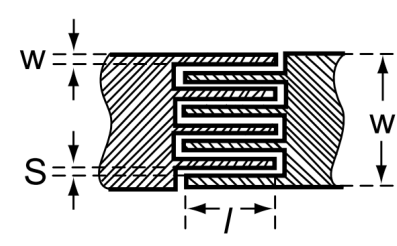

(b)

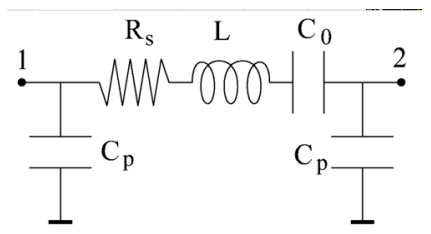

(c)

Fig. 1 (a) Overlay and (b) IDC capacitors configuration and (c) equivalent circuital model of the IDC

The meander inductor (see (a) Fig. 2) as compared with an inductor in the form of a rectangular coil (see (b) Fig. 2) with the same area has a lower inductance and a lower SRF [3 p. 58]. However, the meander can be fabricated on a single conductor layer.

(a)
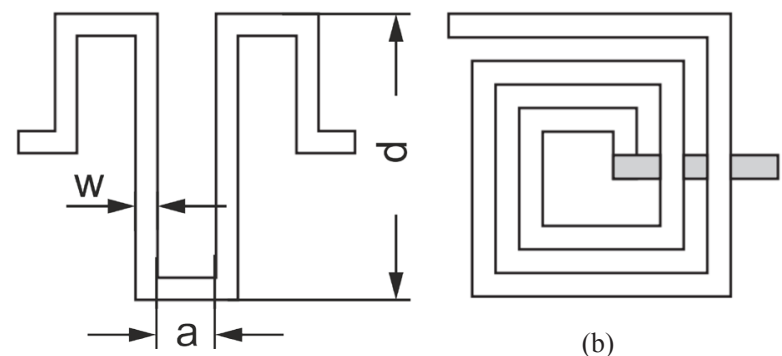

(b)

Fig. 2 Different inductors design: (a) meander and (b) rectangular coil.

Calculation of meander's inductance can be held in accordance with [7]:

$$
L=0.1 \cdot d \cdot\left[4 \cdot n \cdot \ln (2 \cdot(a+w) / w)-K_{n}\right]
$$

where, $d$ - height of the meander, $a$ - the distance between adjacent conductors, $w$ - conductor width, $K_{n}-$ constant.

The resonant circuit by a parallel connection of passive elements (inductor and capacitor) can be done.

It is important to calculate precisely the parameters of passive components for achieving a given device operating frequency of $100 \mathrm{MHz}$. Since Computer-aided engineering (CAE) systems are widely used in design, simulation, and validation of RF devices, COMSOL Multiphysics ${ }^{\circledR}$ have been used for the calculation of capacitance of the different capacitors design.

\section{Experimental}

Passive planar components with different design have been developed in order to quantitatively evaluate the dependence of the electrical parameters on their geometric form.

\subsection{The capacitors geometric configuration}

IDC's capacities changes are possible to achieve with different properties of the substrate i.e. its dielectric constant $\varepsilon_{r}$ and thickness, for a given topology.

However, when design the IDC the parasitic inductance of the electrodes has to be evaluated [8]. IDC can be represented as a simplified series resonant circuit at frequencies below the first IDC's SRF $f_{c}$ (see (c) Fig. 1). The resonant frequency in this case [4 p. 235] is:

$$
f_{c}=1 /(2 \cdot \pi \cdot \sqrt{L \cdot C})
$$

Therefore, to estimate the SRFs' dependence on the electrodes' design of various types of capacitor (see (a) (b) (c) Fig. 3). were examined.
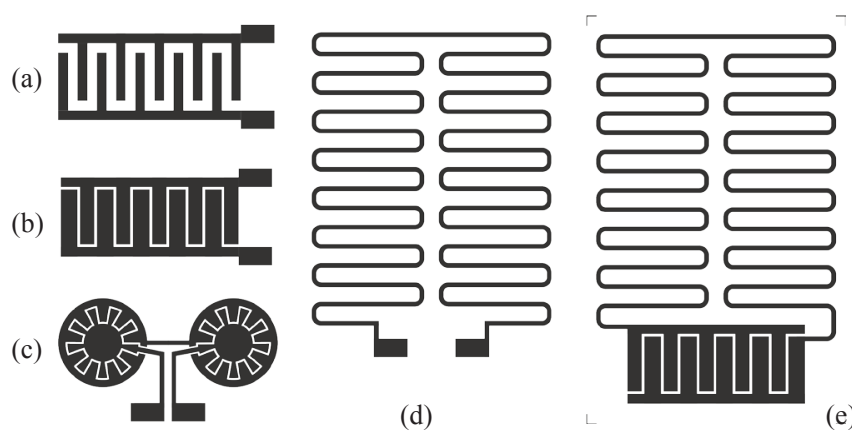

Fig. 3 Design of passive components: capacitors (a, $w=s=2 \mathrm{~mm}$ $l=10 \mathrm{~mm} ; \mathrm{b}, w=3.5 \mathrm{~mm} s=0.35 \mathrm{~mm} l=10 \mathrm{~mm} ; \mathrm{c}, \varnothing=18.5 \mathrm{~mm}$ $s=0.35 \mathrm{~mm}$ ): (d) inductor and (e) resonant circuit.

Equation (1) is applicable for a rectangular IDCs' electrodes and does not take into account the substrate thickness. Therefore, analytically calculate the capacity of the more complex structures (see (c) Fig. 3) is not possible (Table 4).

Therefore, the application COMSOL Multiphysics ${ }^{\circledR}$ has been used to calculate the capacity of capacitors (see Fig. 4 and Table 4).

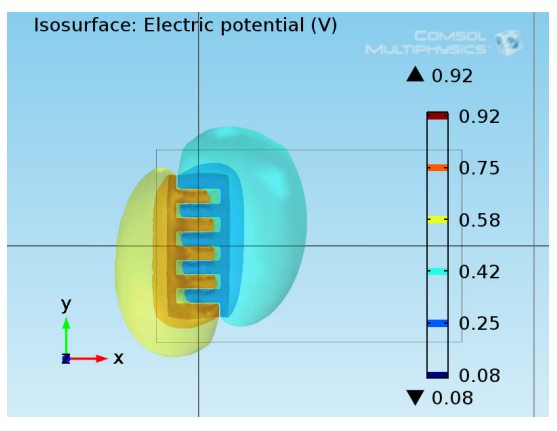

Fig. 4 Equipotential surface of capacitor (b) 
Samples of passive elements in order to test the adequacy of the calculations and built models were produced. The developed models allow to calculate the capacitor capacitance values for a given thickness and the dielectric constant of the substrate. The calculated values of the IDCs' capacitance realized at the FR-4 substrate are listed in Table 4.

\subsection{The inductors geometric configuration}

From it is known that at frequencies below the first meander's SRF the meander's equivalent circuit is a parallel resonant circuit [9] (see Fig. 5).

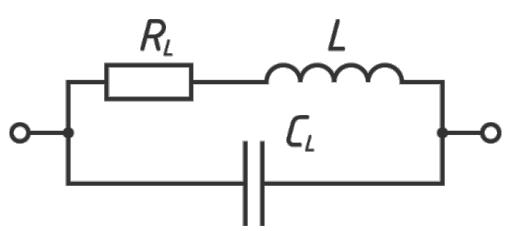

Fig. 5 Equivalent circuit element model of inductor

The geometric configuration of the meander determines parameters such as inductance of the meander, mutual inductance and parasitic capacitance. To ensure stable operation of the parallel resonant circuit (see (e) Fig. 3), meander have to keep its inductive properties at the circuit's resonant frequency, i.e. have a SRF higher than the circuit's resonant frequency. The geometrical dimensions of the meander were calculated based on maximizing the inductance in a given square while minimizing the parasitic capacitance value. Calculated inductance value is $450 \mathrm{nH}$.

\subsection{Manufacture technologies}

Influence of manufacturing processes on RF planar passive components electrical parameters was evaluated by the example of using two technologies: screen printing and photolithography.

Resonant circuit (see (e) Fig. 3), which is located at one conductive layer, can be obtained at a given design of passive elements. This makes it possible to produce samples according to simpler technologies, without an application of additional dielectric layers (in case of screen printing) and vias (in case of photolithography). Reduction in the cost of manufacturing technology is crucial when using the proposed passive elements in the RFID tags.

The quality factor is a major quantitative characteristic of a whole resonant circuit $(Q)$ and of passive components $\left(Q_{L}, Q_{C}\right)$ in particular.

$$
Q_{L}=\left(2 \cdot \pi \cdot f_{o L} \cdot L\right) / R_{L}
$$

For a given layout of conductors and a specific set of substrates' and conductors' materials the quality factor can be optimized by reducing the resistive, inductive, and capacitive losses.

Printing of passive components was provided on semi-automatic printing machine by screen printing technique by one or several layers of printing. Printing was carried out using three different substrates (Table 1): polyethylene terephthalate (PET) film, polyethylene naphthalate (PEN) film, polyimide (PI) film. Silver-containing paste with a resistivity less than $0,003 \Omega / \square$ was used for printing. Printing in several layers was carried out with intermediate drying of each printing layer.

Table 1 Performance comparison for dielectric substrates

\begin{tabular}{lllll}
\hline & FR-4 & PEN & PET & PI \\
\hline Substrate thickness, mm & 1.5 & 0.18 & 0.18 & 0.13 \\
Relative permittivity & 5 & 2.9 & 3.3 & 3.5 \\
\hline
\end{tabular}

Samples fabricated by photolithography on $60 \mu \mathrm{m}$ thick polycarbonate film with a less than $1 \mu \mathrm{m}$ copper layer and on $1.57 \mathrm{~mm}$ thick FR-4 substrate with an $18 \mu \mathrm{m}$ copper layer was also investigated. Due to the high resistivity value $(2 \Omega / \square)$ inductors on polycarbonate film were unworkable (see Table 2).

\section{Results}

The frequency characteristics of the parameters (inductance, capacitance, quality factor, impedance $Z$ ) of planar passive components were carried out by the RF RLC meter Agilent 4287A in the frequency range from $1 \mathrm{MHz}$ to $500 \mathrm{MHz}$.

\subsection{Inductor measurement}

Along with the inductance, quality factor and self-resonant frequency of the inductors are the most important parameters that were measured. Determination of inductors' SRF was made based on the frequency dependence of the impedance.

The meanders' DC resistance were measured to evaluate the resistance losses values (Table 2). At this stage of the measurement, it was found, that due to the small thickness of the copper conductive layer meander made by photolithography method on a polycarbonate film has large resistance losses.

Table 2 Basic parameters of inductors

\begin{tabular}{|c|c|c|c|c|c|c|}
\hline \# & & $\begin{array}{l}\mathrm{R}_{\mathrm{s}} \\
\text { [ohms] }\end{array}$ & $\begin{array}{l}\mathrm{L} \\
{[\mathrm{nH}]}\end{array}$ & $\begin{array}{l}\text { Quantity of } \\
\text { layers of the } \\
\text { print }\end{array}$ & $\begin{array}{l}\text { Conductors } \\
\text { thickness }[\mu \mathrm{m}]\end{array}$ & Substrate \\
\hline 1 & \multirow{7}{*}{ 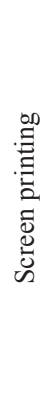 } & 77 & 418 & 1 & 13 & PET \\
\hline 2 & & 34 & 424 & 2 & 26 & PET \\
\hline 3 & & 20 & 438 & 3 & 38 & PET \\
\hline 4 & & 70 & 424 & 1 & 12 & PEN \\
\hline 5 & & 36 & 432 & 2 & 25 & PEN \\
\hline 6 & & 72 & 420 & 1 & 13 & PI \\
\hline 7 & & 35 & 431 & 2 & 30 & PI \\
\hline 8 & \multirow{2}{*}{$\begin{array}{l}\frac{\dot{\Xi}}{0} \\
\frac{0}{0} \\
\frac{0}{0}\end{array}$} & 2 & 419 & - & 18 & FR-4 \\
\hline 9 & & 1120 & - & - & less than $1 \mu \mathrm{m}$ & $\mathrm{PC}$ \\
\hline
\end{tabular}


It is generally known that the inductance is determined by the geometrical parameters of the inductor, which confirms the Eq. (2). This is also shown by the results of measurements (Fig. 5). Where is shown that inductance is practically independent of the conductor and of the type of substrate at frequencies up to $100 \mathrm{MHz}$. Inductance values at $1 \mathrm{MHz}$ do not differ by more than 5\% and not more than $7 \%$ at $100 \mathrm{MHz}$. Also the highest frequency dependence has a meander formed on the FR-4 substrate.

The inductors' quality factor depends on the type of conductor and it is essentially independent of the substrate (3). $Q_{L}$ reaches the highest values at the frequency range from $80 \mathrm{MHz}$ to $100 \mathrm{MHz}$ of 82 for the copper meander and 5.9 and 2.9 for 3 or 1-layer printed meanders (Fig. 6). The $Q_{L}$ values for printed inductors proportionally relate to their resistance measurements. Resistance of inductors decreases proportionally with increasing the number of printing layers (Table 2).

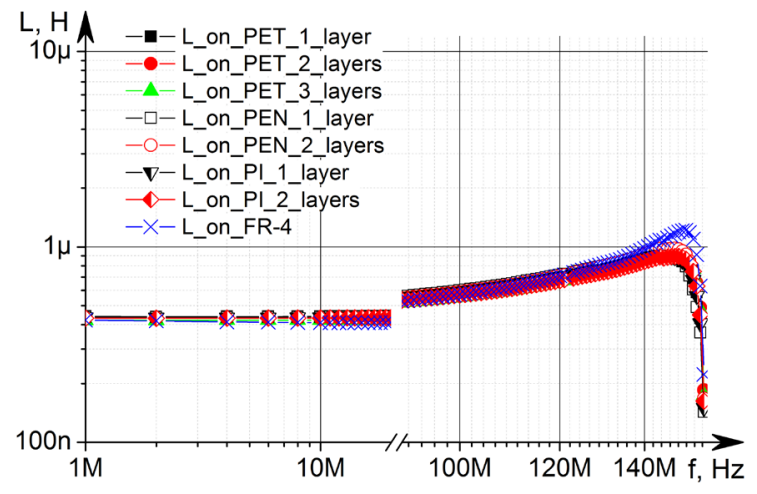

Fig. 6 Inductance of the inductors

The inductor's SRF shows at what frequency reactance of the inductance and parasitic capacitance become equal. The parasitic capacitance increases with the dielectric constant and thickness of the material. The lowest measured value of the SRF have the inductor implemented on a FR-4 substrate (176 MHz). Printed inductors SRF are between $179 \mathrm{MHz}$ (for PI-substrate) and $182 \mathrm{MHz}$ (for PEN-substrate) and weakly depends on the number of printing layers. The obtained SRF values are correlated with the data about the thickness and dielectric constant of the substrate materials (Table 1).

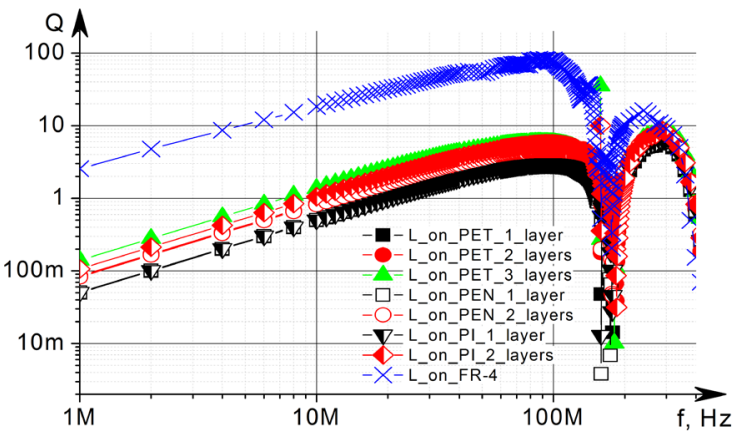

Fig. 7 Quality factor to the frequency of the inductors

\subsection{Capacitors measurement}

For IDC its capacitance, SRF and the quality factor $Q_{C}$ were measured. IDC capacitance measurements showed that for each form holds that the IDC capacitance increases more than 1.5 times when implementation of FR-4 substrate, as compared with printing on PET. Also, increasing the number of printing layers increases the IDC capacity (up to $15 \%$ for 3 -layer printing) (Table 3).

Reducing the gap between the electrodes (capacitors (a) and (b) on Fig. 2) increases the IDC capacitance (Table 3).

Changing the geometric form of the capacitors' electrodes and the gap between them helps to reduce capacitors' own parasitic inductance, which in turn leads to higher self-resonant frequency (Fig. 8). At comparable values of capacitance and the capacitors area, a reduction of capacitors SRF by changing the capacitors geometric form can be obtained [10].

Table 3 Comparison of the IDCs capacitance

\begin{tabular}{l|lll}
\hline \multirow{2}{*}{ Capacitance, $\mathrm{pF}$} & \multicolumn{3}{|c}{ Capacitors type (according to Fig. 2) } \\
\cline { 2 - 4 } & $\mathrm{a}$ & $\mathrm{b}$ & $\mathrm{c}$ \\
\hline SP on PET 1 layer & 1.94 & 3.60 & 1.73 \\
SP on PET 2 layers & 2.03 & 3.95 & 1.75 \\
SP on PET 3 layers & 1.94 & 4.23 & 1.97 \\
SP on PEN 1 layer & 2.31 & 3.91 & 1.84 \\
SP on PEN 2 layers & 1.72 & 3.86 & 1.82 \\
SP on PI 1 layer & 1.94 & 3.45 & 1.32 \\
SP on PI 2 layers & 1.85 & 3.55 & 1.86 \\
Photolithography on FR-4 & 3.72 & 7.06 & 3.02 \\
Photolithography on PI film & 1.54 & 0.95 & 1.13 \\
\hline
\end{tabular}

The quality factor values of capacitors implemented on the FR-4 substrate are at the level of 100 . The highest quality factor (300) has capacitors, printed on the PI film.

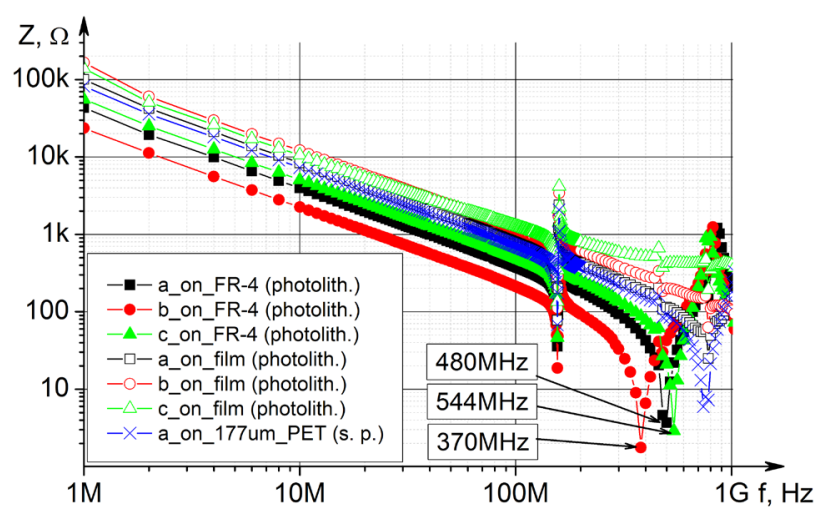

Fig. 8 Impedance to the frequency characteristic of the capacitors 
Table 4 Capacitance measurements at $1 \mathrm{MHz}$

\begin{tabular}{l|lcc}
\hline & Capacitance & \multicolumn{4}{|c}{ Capacitors type (according to Fig. 2) } \\
\cline { 2 - 4 } & $\mathrm{a}$ & $\mathrm{b}$ & $\mathrm{c}$ \\
\hline Results of measurements at $1 \mathrm{MHz}$ & $3.72 \mathrm{pF}$ & $7.06 \mathrm{pF}$ & $3.02 \mathrm{pF}$ \\
Results of simulation & $3.62 \mathrm{pF}$ & $7.93 \mathrm{pF}$ & $5.00 \mathrm{pF}$ \\
Analytical calculation & $3.50 \mathrm{pF}$ & - & - \\
\hline
\end{tabular}

\subsection{Measurements of resonant circuits}

Comparison of high-frequency properties of resonant circuits was carried out based on the resonance frequency and the quality factor measurements.

Screen printing technology allows to get resonant circuits with a similar value (about $108 \mathrm{MHz}$ ) of the resonant frequency despite the application of three types of substrates (Fig. 9). However, large number of layer of print increases quality factor $Q$ of the circuit from 4 with a single-layer printing to 6.5 during the three-layer printing. The quality factor FR-4 circuit was greatest $(Q=40)$ due to much smaller conduction losses.

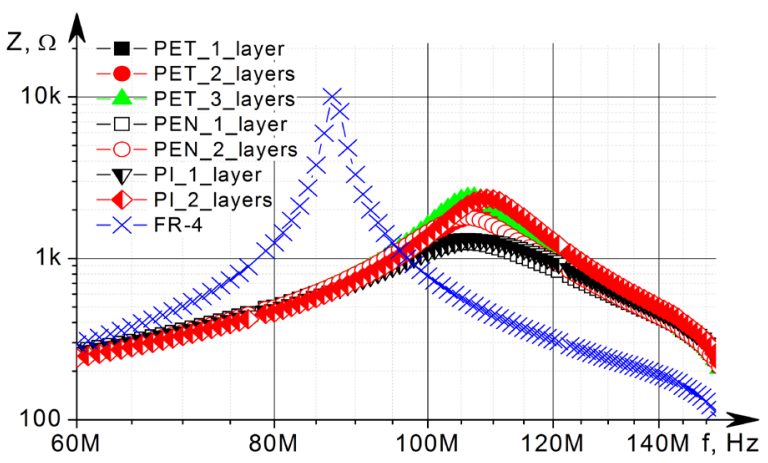

Fig. 9 Frequency characteristics of parallel resonant circuits (d)

The passive components SRFs allow achieving the stable resonant frequency of the circuits.

The difference between the results of measurements (88 MHz, see Fig. 10) and calculations of the resonant frequency $(112 \mathrm{MHz})$ of the resonant circuit implemented on FR-4 substrate does not exceed several tens of $\mathrm{MHz}$ due to multiplication of inaccuracies in calculated $C$ and $L$ values.

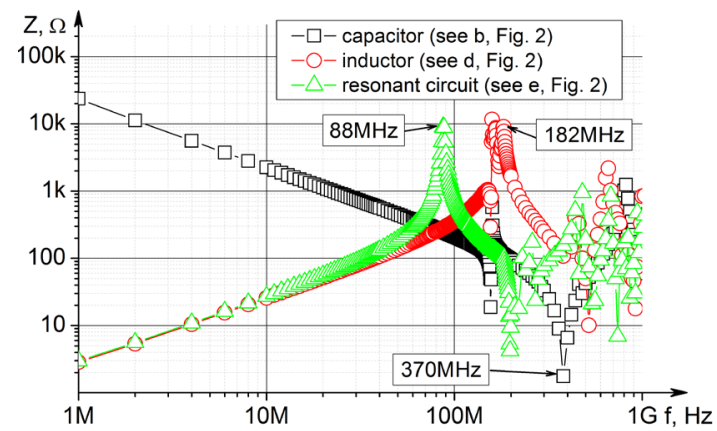

Fig. 10 Impedance to the frequency characteristic of the resonant circuit and passive components implemented on the FR-4 substrate

\section{Discussions}

The measurement results showed the manufacturing technology dependence on passive components parameters. And for capacitors and inductors are various critical factors. This is due to the physical principles of passive elements.

Measurements have shown the inductors $Q_{L}$ value from 3 to 40 . This parameter is directly dependent (3) on series resistance of the conductor. At high frequencies, the series resistance is significantly increased due to the effects of the skin effect. This reduces the quality factor $Q_{L}$. Thus the $Q_{L}$ of all inductors reaches a maximum at frequencies up to $110 \mathrm{MHz}$. With screen printing layers increasing there is a proportional decrease in the resistance (Table 2). This in turn leads to an $Q_{L}$ increase from 3 to 6 . Since the inductor on FR-4 has a significantly lower resistance, the $Q_{L}$ value reaches 40 .

Also, for the screen printing meanders, different substrate materials do not have a noticeable effect on inductors parasitic capacitance on which depends SRF. Differences for the SRF do not exceed $3 \%$ in this case. This may be explaining by the small difference in the thickness and relative permittivity of the substrates. The resonant frequency of a meander on the FR-4 in this case is fewer on $5 \mathrm{MHz}$. This SRF decrease can be explained by more than 8 times greater thickness value of the substrate and the larger value of $\varepsilon_{r}$.

Capacitance of the capacitor can be changed from $15 \%$ (for screen printing) to more than $200 \%$ (for FR-4) by replacing the substrate material for the same shape of the electrodes (Table 4). There are also differences in the comparison of capacitors with different shapes of electrodes on the same substrate. Reducing the gap between the electrodes allows to obtain a strong electric field, and as a consequence, higher charge accumulation, which determines capacitance. Electrodes of various forms have different parasitic inductance. Larger parasitic inductance has narrow straight sections of the conductors. Capacitor reduces its SRF in the case of increasing the value of capacitor's parasitic inductance. If the first SRF is less than the device operating frequency, then capacitor lose its own capacitive performance. The studied capacitors (see (a) (b) (c) Fig. 3) produced by screen printing technology have for each topology its own SRF, each of which is within 3\% of nominal for a given topology. Similar forms of capacitors on FR-4 have smaller SRF values that can be attributed to large values of capacitance (since SRF is determined by the values of capacitance and parasitic inductance of the circuit (c) in Fig. 1). SRF of all forms of capacitors made by any of the described technology is much more than a given operating frequency in $100 \mathrm{MHz}$. Therefore, when choosing the method of manufacturing of the resonant circuit, can be taken into consideration only the capacitance value and the desired mechanical properties of the substrate.

Assessment of the resonant circuit operation stability was carried out by comparing passive elements SFRs to the resonant frequency of the oscillatory circuit. On the graph is possible to 
define the bandwidth at 0.707 amplitude value of the impedance (minimum - for the capacitor and the maximum - for the inductor). The efficiency of the circuit ensured in the absence of overlays bandwidth of a passive element with a given resonant frequency [11].

The resonance circuit have the stable operating resonant frequency. But the screen printing technology is applicable for the manufacture of single-layer resonant circuits in the case when a product does not need stringent electrical requirements (maximum obtained $Q$ value is less than 5). The same resonant circuit made on FR-4 substrate has $Q=40$.

\section{Conclusions}

The values of meander inductance and IDC capacitance calculated analytically and the values of IDC capacitance calculated using COMSOL Multiphysics ${ }^{\circledR}$ insignificantly differ from the measurements data. This can be explained that the analytical calculations do not take into account the frequency dependence of relative permittivity. Based on the results of the software simulation and measurement of passive elements with standard topologies the value of the electrical parameters at a given frequency for more complex forms of passive elements can estimated.

The advantage of the developed layout of passive elements is the possibility of their production in a single conductive layer, which also increases reliability compared to multilayer technology devices.

Research has shown the manufacturing technology dependence of the passive component's electrical parameters.

IDC capacitance depends on the topology, the permittivity, and the thickness of the substrate material. The greatest capacitors capacity is manufactured on FR-4 substrate. It is possible to enlarge IDC's SRF while maintaining the capacity value through a topology modifying.

Inductors manufacturing process are most affects on their quality factor.

The passive components self-resonant frequency allows achieving the stable resonant frequency of the circuits. At the same time various technologies provide different value of circuits (see (e) Fig. 3) quality factor.

Photolithography on the film does not allow to obtain workable resonant circuit because of the high conductor resistance.

\section{Acknowledgement}

This research has been supported by the Ministry of Education, Youth and Sports of the Czech Republic under the RICE - New Technologies and Concepts for Smart Industrial Systems, project No. LO1607 and by the Technology Agency of the Czech Republic under the FLEXPRINT - project No. TE01020022.

\section{References}

[1] Chang, K., Hsieh, L.-H. "Microwave ring circuits and related structures." John Wiley \& Sons, Inc., Hoboken, New Jersey. 2004.

[2] Chen, Y., He, W., Zhou, G., He, X., Zhou, H., Mo, Y., He, B. "Compaction uniformity and environmental adaptability of printed RFID antenna." In: 2010 International Conference on Anti-Counterfeiting, Security and Identification, Chengdu, July 18-20, 2010, pp. 277-280. https://doi.org/10.1109/ICASID.2010.5551339

[3] Rivadeneyra, A., Fernández-Salmerón, J., Agudo-Acemel, M., LópezVillanueva, J. A., Capitan-Vallvey, L. F., Palma, A. J. "Printed electrodes structures as capacitive humidity sensors: A comparison." Sensors and Actuators A: Physical. 244, pp. 56-65. 2016. https://doi.org/10.1016/j.sna.2016.03.023

[4] Kim, Y., Kim, H., Yoo, H. J. "Electrical Characterization of Screen-Printed Circuits on the Fabric." IEEE Transactions on Advanced Packaging. 33(1), pp. 196-205.2010. https://doi.org/10.1109/TADVP.2009.2034536

[5] Bahl, I. J. "Lumped Elements for RF and Microwave Circuits." Artech House, Norwood, MA. 2003.

[6] Maloratsky, L. G. "Passive RF \& Microwave Integrated Circuits." Newnes, Burlington, MA. 2003

[7] Wadell, B. C. "Transmission Line Design Handbook." pp. 412-414. Artech House, Inc., Norwood, MA. 1991.

[8] Mousavi, S. H., Kouki, A. B. "High-SRF VHF/UHF Lumped Elements in LTCC." IEEE Microwave and Wireless Components Letters. 25(1), pp. 25-27. 2015. https://doi.org/10.1109/LMWC.2014.2369952

[9] Passos, F., Fino, H., Roca, E., Gonzalez-Echevarria, R., Fernandez, F. V. "Lumped element model for arbitrarily shaped integrated inductors - A statistical analysis." In: 2013 IEEE International Conference on Microwaves, Communications, Antennas and Electronic Systems (COMCAS 2013). Tel Aviv, Oct. 21-23, 2013 , pp. 1-5.

https://doi.org/10.1109/COMCAS.2013.6685307

[10] Munir, A., Sari, E. K. "Printed traveling wave antenna composed of interdigital capacitor structure for wireless communication application." In: 2015 International Seminar on Intelligent Technology and Its Applications (ISITIA), Surabaya, May 20-21, 2015 , pp. 441-444. https://doi.org/10.1109/ISITIA.2015.7220022

[11] Napieralski, A. "Preface." In: 2013 Proceedings of the 20th International Conference Mixed Design of Integrated Circuits and Systems MIXDES 2013 : Gdynia, Poland, 20-22 June 2013. pp. 3-4. 